\title{
Photopolarimetric activity and circumstellar environment of the young binary system DF Tau ${ }^{\star, \star \star}$
}

\author{
D. Shakhovskoj ${ }^{1}$, V. Grinin ${ }^{1,4}$, A. Rostopchina ${ }^{1}$, D. Schert1 ${ }^{2}$, K.-H. Hofmann ${ }^{2}$, G. Weigelt ${ }^{2}$, Yu. Balega ${ }^{3}$, \\ O. Kiyaeva ${ }^{4}$, and S. Melnikov ${ }^{5}$
}

${ }^{1}$ Crimean Astrophysical Observatory, Crimea, 334413 Nauchny, Ukraine

e-mail: grinin@crao.crimea.ua

2 Max-Plank Institute fur Radioastronomie, Bonn, Germany

e-mail: weigelt@mpifr-bonn.mpg.de

3 Special Astronomical Observatory, Russia

4 Pulkovo Astronomical Observatory, St. Petersburg 198904, Russia

e-mail: grinin@vg1723.spb.edu

5 Astronomical Institute, Tashkent, Uzbekistan

Received 28 July 2005 / Accepted 27 October 2005

\section{ABSTRACT}

We present the results of multi-year photometric and polarimetric observations of the young binary system DF Tau in the UBVRI bands, as well as bispectrum speckle interferometric observations in the $H$ and $K$ bands obtained between 2001 and 2003. The photometric and polarimetric observations suggest that the linear polarization of DF Tau does not depend on its brightness, and the polarization variation has a stochastic character. This result confirms earlier suggestions about the dominant role of hot accretion spots in the photometric activity of this star. We argue that the hot spots are at high latitudes and/or the star rotation axis is inclined to the line-of-sight. The influence of circumstellar (CS) dust on the variability is probably small since the inclination of the primary's CS disk to the line-of-sight is large.

Using the total mass of the binary system DF Tau from Hartigan \& Kenyon (2003, ApJ, 583, 334), we calculated new orbital parameters of the system. The new value of the orbital period ( $P=74.1 \mathrm{yr}$ ) disagrees with the photometric cycle of about $40 \mathrm{yr}$ revealed by Lamzin et al. ( $2001 \mathrm{~b}$, A\&A, 372, 922) from analysis of the historical light curve of this star. This suggests that this cycle is not caused by the orbital motions of the binary components, but probably reflects the cyclical variability of the global magnetic field on the main component.

Key words. techniques: photometric - stars: pre-main sequence - techniques: interferometric - techniques: polarimetric binaries: general stars: individual: DF Tau

\section{Introduction}

DF Tau is one of the most active classical T Tauri stars (CTTS) (Herbig \& Bell 1988). It exibits a strong and irregular brightness variability at optical wavelengths (Zaitseva \& Lyutyi 1976; Rydgren et al. 1984; Walker 1987; Bouvier et al. 1988) that is attributed to compact accretion hot spots that originate on the stellar surface at places where accretion matter comes in contact with the stellar atmosphere (e.g. Grinin 1980; Bouvier $\&$ Bertout 1989). The existence of hot spots on a rotating star should lead to the appearance of sinusoidal waves on the light curve of the star (Bouvier et al. 1986; Bouvier \& Bertout 1989). In general, however, the light curve of DF Tau, like the

^ Based on data collected at the Crimean Astrophysical Observatory (CrAO), Maidanak Observatory, and with the $6 \mathrm{~m}$ telescope of the Special Astrophysical Observatory.

$\star \star$ Table 1 is only available in electronic form at the CDS via anonymous ftp to cdsarc.u-strasbg.fr $(130.79 .128 .5)$ or via http://cdsweb.u-strasbg.fr/cgi-bin/qcat?J/A+A/448/1075 light curves of other CTTSs, displays aperiodic light variability. The continuum radiation of the hot spots is the source of the veiling observed in the spectrum of DF Tau, as well as in the other CTTSs (Basri \& Batalha 1990; Hartigan et al. 1991; Johns-Krull \& Basri 1997; Gullbring et al. 1998; Chelli et al. 1999). The variability of the accretion rate, in combination with the geometric changes of the accretion spots due to the star rotation leads to the spectral variability observed in almost all lines (Johns \& Basri 1995; Johns-Krull \& Basri 1997; Unruh et al. 1998).

In the binary system DF Tau (Chen et al. 1990), the secondary is a low mass M V star (Ghez et al. 1997; White \& Ghez 2001). Due to the small separation, they cannot be resolved by photometric observations with ground-based telescopes. From observations with the Hubble Space Telescope (HST), White \& Ghez (2001) find that the contribution of the companion to the optical spectrum of the system decreases at $\lambda<6000 \mathrm{~A}$ and is less than $10 \%$ at $\lambda \approx 3000 \mathrm{~A}$. Since the variability amplitude 
of DF Tau increases at short wavelengths, this suggests that the primary is the main source of the variability (Ghez et al. 1997). This was then confirmed by direct photometric observations of DF Tau with the HST, where Schaefer et al. (2003) show that at the brightness minima, the primary is only slightly brighter $(V \approx 13.1)$ than the secondary $(V \approx 13.5)$.

Recently, Tamazian et al. (2002) have summarized all of the published speckle interferometric observations of DF Tau made until 1998 and derived a preliminary orbit and system parameters. According to their data, the orbital period of the system is $P=92.8 \pm 5 \mathrm{yr}$. However, Schaefer et al. (2003) have made new measurements of the orbital motions of DF Tau with the Fine Guidance Sensors of the HST and have shown that the orbital parameters of DF Tau, including its period, are still quite uncertain. In particular, the value of the orbital period might be in the range of 50 to $150 \mathrm{yr}$, according to their calculations. Additional speckle interferometric observations are needed to improve the orbital parameters.

According to Kenyon et al. (1994), Johns-Krull \& Basri (1997), Chelli et al. (1999), and Lamzin et al. (2001a), the spectroscopic activity of DF Tau can be described in the framework of the magnetospheric accretion model. Chelli et al. (1999) have shown that several episodes of its photometric activity may be the result of the variable occultation of the star by optically thick CS clouds.

There are only a few single observations of DF Tau's linear polarization available at present (Bastien 1982, 1985; Menard \& Bastien 1992). Simultaneous photometry and polarimetry of young stars are an important tool for investigating the activity of young stars (see review by Grinin (2000) and references therein). In particular, as was shown by Wood et al. (1996) and Stassun \& Wood (1999), a young star with hot spots surrounded by a dusty disk is a polarimetrically active object: its linear polarization should be stochastic due to the unsteady accretion. Besides, it can anti-correlate at certain conditions when the brightness changes.

In this paper, we analyze the results of the simultaneous, multi-year $U B V R I$ photometric and polarimetric observations of DF Tau made at the Crimean Astrophysical Observatory (CrAO), the results of photometric $U B V R$ monitoring of this star made at the Maidanak Observatory, as well as near-infrared bispectrum speckle interferometry obtained with the $6 \mathrm{~m}$ telescope of the Special Astrophysical Observatory (SAO). The goal of this analysis is to combine the data from the different sources in order to study the accretion activity of this young binary star.

\section{Observations and data reduction}

\subsection{Photometric and polarimetric observations}

Photometric and polarimetric observations of DF Tau were carried out between 1991 and 1998 using the five-band photopolarimeter designed by Piirola (1984) and mounted at the $1.25 \mathrm{~m}$ telescope of the Crimean Astrophysical Observatory ( $\mathrm{CrAO})$. This instrument permits simultaneous observations of polarization and brightness in the $U, B, V, R$, and $I$ bands. The effective wavelengths of the instrumental system are close to

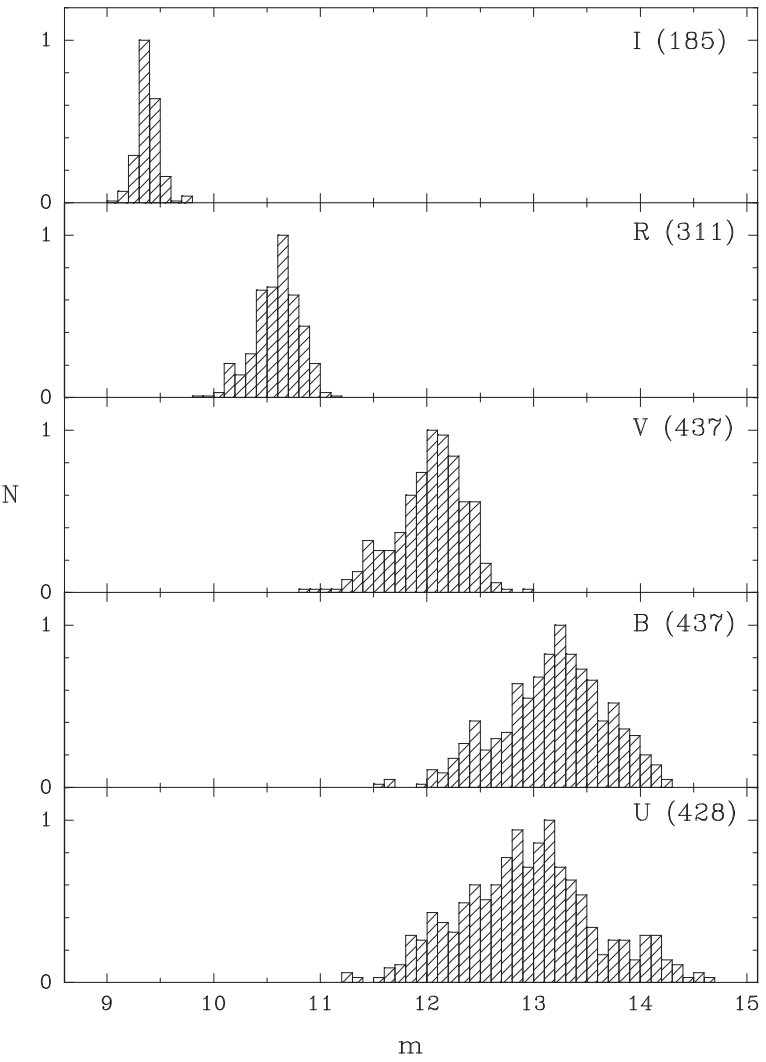

Fig. 1. Histogram of the photometric activity of DF Tau based on the photometric data of different authors (see text).

the standard wavelengths of the Johnson photometric system. We reduced our observations using observations of comparison stars. We generally used a diaphragm with a $10^{\prime \prime}$ diameter, although a $15^{\prime \prime}$ diaphragm was used during nights with poor seeing conditions. We averaged the photometric observations acquired on a single night. The mean photometric uncertainty was approximately $0.03^{\mathrm{m}}$ in the $U$ band and $0.01^{\mathrm{m}}$ in all other bands.

A vast majority of the photopolarimetric observations of DF Tau were obtained in the simultaneous photometry and polarimetry mode. Depending on the observational conditions (weather, the star's brightness), each observation of linear polarization consisted of 12 to 36 measurements of the Stokes parameters. Corrections for instrumental polarization and the system's zero point were determined each month using observations of standard stars. The results of our observations are presented in Table 1 and in Figs. 1, 3-7.

In our analysis, we also used the photometric $U B V R$ data from the catalogue by Herbst et al. (1994). The main part of the data was obtained at the Maidanak Observatory between 1984 and 2000 and was completed with the new observations of DF Tau (from 2001 to 2003) from the photometric data base of this observatory.

\subsection{Bispectrum speckle interferometry}

Speckle interferograms of DF Tau were recorded with the SAO $6 \mathrm{~m}$ telescope in 2001, 2002, and 2003. The detector of our 
Table 2. Speckle observations. $\lambda_{\mathrm{c}}$ and $\Delta \lambda$ give the central wavelength and bandwidth of the filter. $N_{\mathrm{O}}$ and $N_{\mathrm{R}}$ are the numbers of speckle interferograms of object and reference-star, respectively. $T$ is the exposure time per frame and $S$ is seeing (FWHM). In the last column, the names of the reference stars are given.

\begin{tabular}{lllllllll}
\hline \hline Epoch & $\begin{array}{l}\lambda_{\mathrm{c}} \\
{[\mathrm{nm}]}\end{array}$ & $\begin{array}{l}\Delta \lambda \\
{[\mathrm{nm}]}\end{array}$ & $\begin{array}{c}\text { Pixel size } \\
{[\mathrm{mas}]}\end{array}$ & $N_{\mathrm{O}}$ & $N_{\mathrm{R}}$ & $\begin{array}{c}T \\
{[\mathrm{~ms}]}\end{array}$ & $\begin{array}{l}S \text { Reference } \\
{\left[{ }^{\prime \prime}\right]}\end{array}$ & Star \\
\hline 2001.92 & 1648 & 317 & 20.08 & 695 & 565 & 160 & 2.3 & HIP 19186 \\
2002.74 & 2115 & 214 & 27.02 & 924 & 910 & 184 & 2.1 & HIP 18629 \\
2003.85 & 2115 & 214 & 28.73 & 461 & 436 & 218 & 2.0 & HD 283655 \\
\hline
\end{tabular}

Table 3. Results of speckle observations of DF Tau.

\begin{tabular}{ccllc}
\hline \hline Data & Band & $\begin{array}{l}\rho \\
\text { mas. }\end{array}$ & $\begin{array}{l}\theta \\
\text { deg. }\end{array}$ & $\begin{array}{c}\text { Int. Ratio. } \\
q\end{array}$ \\
\hline 2001.92 & $H$ & $104 \pm 2$ & $260.2 \pm 1$ & $0.76 \pm 0.02$ \\
2002.74 & $K$ & $105 \pm 3$ & $256.0 \pm 2$ & $0.61 \pm 0.02$ \\
2003.85 & $K$ & $109 \pm 3$ & $252.5 \pm 2$ & $0.50 \pm 0.02$ \\
\hline
\end{tabular}

speckle camera was a $512 \times 512$ pixel Rockwell HAWAII array. Further observational parameters are listed in Table 2 . The object power spectrum was determined with the speckle interferometry method (Labeyrie 1970). Speckle interferograms of unresolved single stars were recorded just before and after the observations of the object and were used as reference stars for the determination of the speckle interferometry transfer function. Images of DF Tau were reconstructed using the bispectrum speckle interferometry method (Weigelt 1977; Weigelt \& Wirnitzer 1983; Lohman et al. 1983; Hofmann \& Weigelt 1986).

The results are presented in Table 3 and in Figs. 8 and 9.

\section{Results}

\subsection{Photometric activity}

The histograms of DF Tau's photometric activity presented in Fig. 1 are based on the data obtained at Maidanak and $\mathrm{CrAO}$, as well as the data of the other groups from the Herbst et al. (1994) catalogue. Each observation in the histogram corresponds to one photometric measurement per night. For nights when several measurements were made, the mean result was used. One can see that the amplitude of the photometric activity increases as the wavelengths decrease, which is typical of $\mathrm{T}$ Tauri stars. The photometric histograms of the different pass bands have different shapes. In the $I, R$, and $V$ bands, the shapes are typical of flare stars (Parenago 1956): the "tails" of the histograms on the bright side demonstrate that energetic flares are rare events. The $U$ and $B$ histograms are more symmetric, indicating that the weakest states of the star in the blue are especially rare.

From the light curve presented in Fig. 4, one can see that the star showed irregular optical variability that sometimes has the form of the flare-like event observed at $\mathrm{JD} \approx 2450004$. Its amplitude decreased with increasing wavelength: $\Delta U=$ 0.97, $\Delta B=0.95, \Delta V=0.66, \Delta R=0.29, \Delta I=-0.07$. In

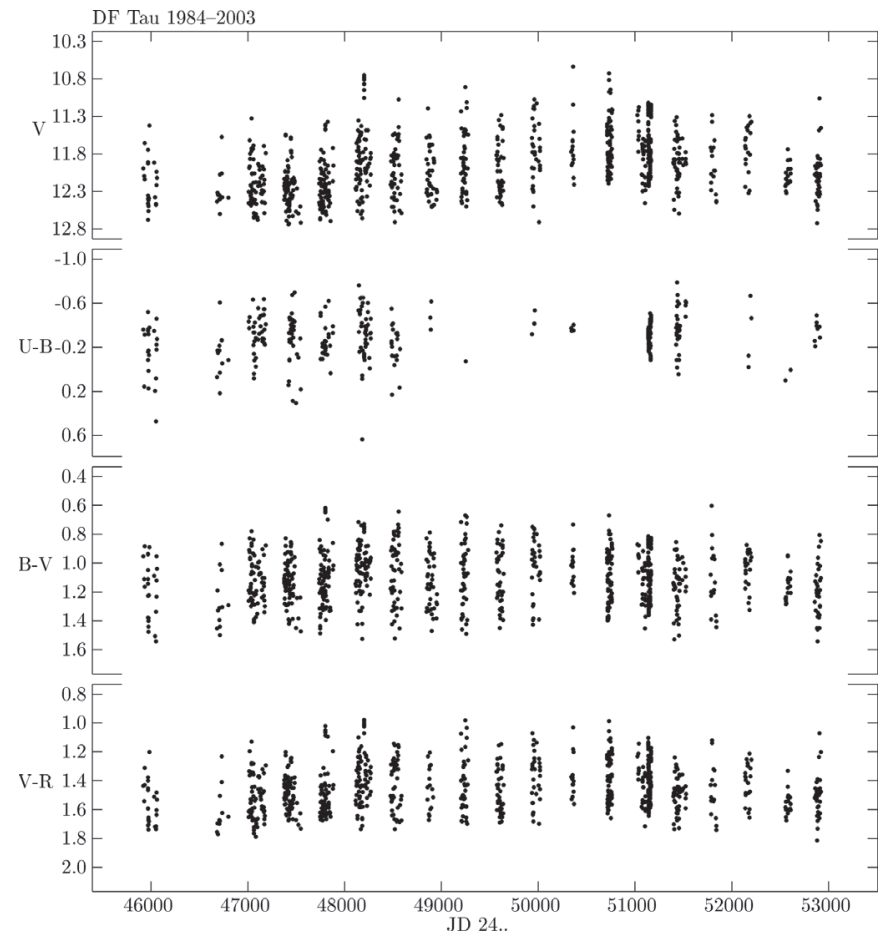

Fig. 2. Light curve of DF Tau in $V$ band and the color indexes $U-B$, $B-V$, and $V-R$ based on the Maidanak observations.

the past, similar flare-like events in DF Tau were observed (see e.g. Zaitseva \& Lyutyi 1976; Rydgren et al. 1984).

An interesting feature of the event observed at JD 2450004 (Figs. 4 and 5) is that the star did not return to its pre-flare state after the flare decay. Instead, the stellar flux decreased to well below the pre-flare state. The amplitudes of the post-flare minimum increased to short wavelengths: $\Delta U=2.03, \Delta B=$ 1.75, $\Delta V=1.19, \Delta R=0.66, \Delta I=0.32$. A similar episode in the photometric activity of DF Tau was observed earlier by Zaitseva \& Luyty (1976), but on a much shorter (a few hours) time scale and with a smaller amplitude. We discuss the possible origin of such unusual events below, after discussing of the polarimetric behavior of the star.

Brightness changes of DF Tau are accompanied by color variations (Figs. 2 and 3): the star becomes bluer when its brightness increases (Fig. 3). Similar variations in the color indices were observed earlier (see e.g. Herbst et al. 1994, and references therein). In the framework of the model of surface accretion activity, these color variations can be explained by variations of the gas temperature in the hot accretion spots on the stellar surface, which reach the maximal values near the brightness maxima. The continuum radiation of the hot spots in these phases (including free-bound and free-free radiation of $\mathrm{H}$ and $\mathrm{H}^{-}$) is a quasi-black body, and its color corresponds to a gas temperature on the order of $10^{4} \mathrm{~K}$ (Grinin 1980).

It is interesting to note that, according to the colormagnitude diagrams (Fig. 3), the minimal values of the $B-V$ color index were observed at the light maxima of DF Tau, while the minimal values of the $U-B$ color index were observed in the intermediate brightness states. Such a non-monotonic change of the $U-B$ index follows from the 

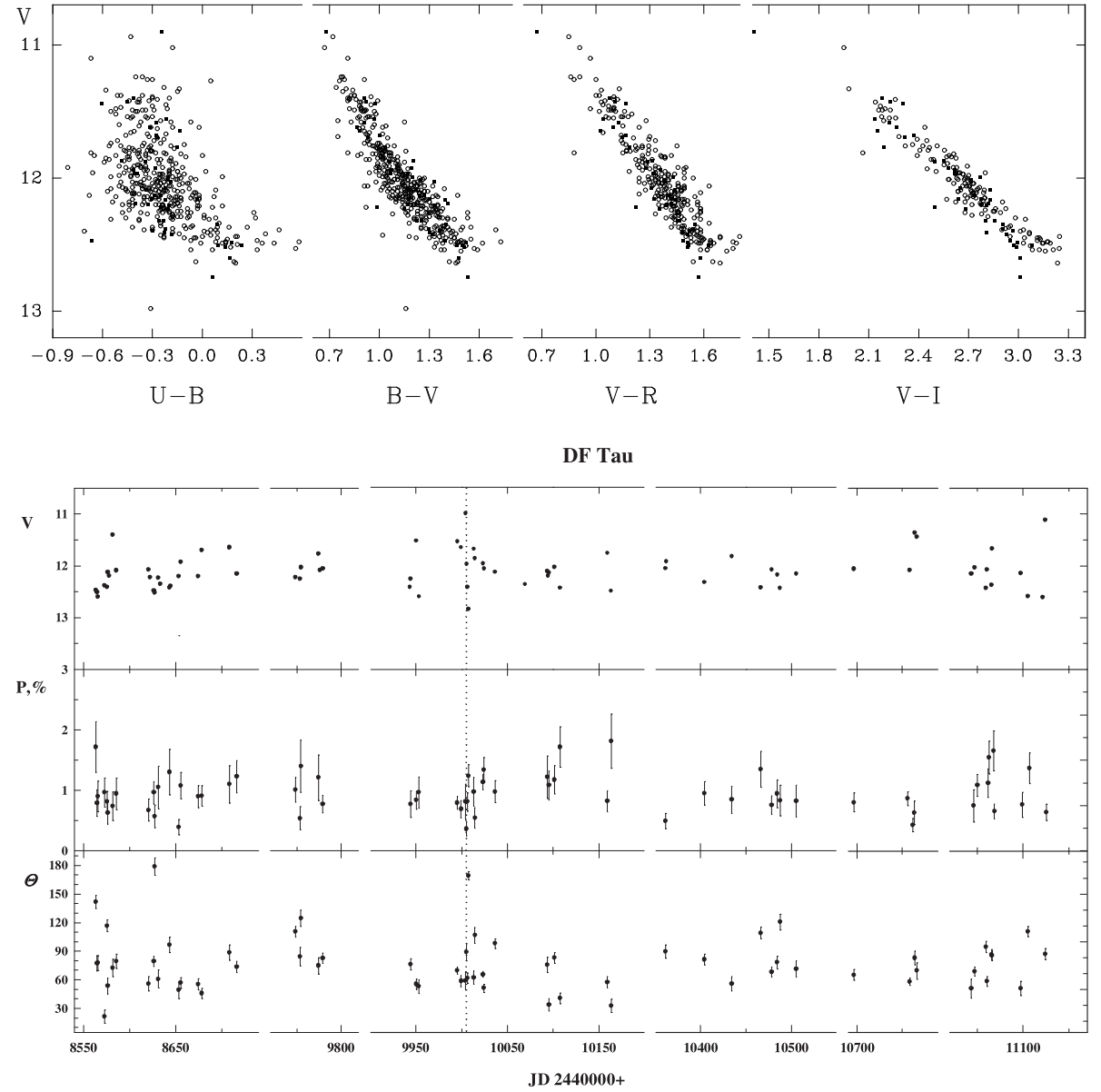

Fig. 3. Color-magnitude diagram of DF Tau. Points: the Crimean photometric data; open circles: the data from the catalog by Herbst et al. (1994).

Fig. 4. $V$-magnitude, degree, and position angle of the linear polarization of DF Tau based on the Crimean photo-polarimetric data.

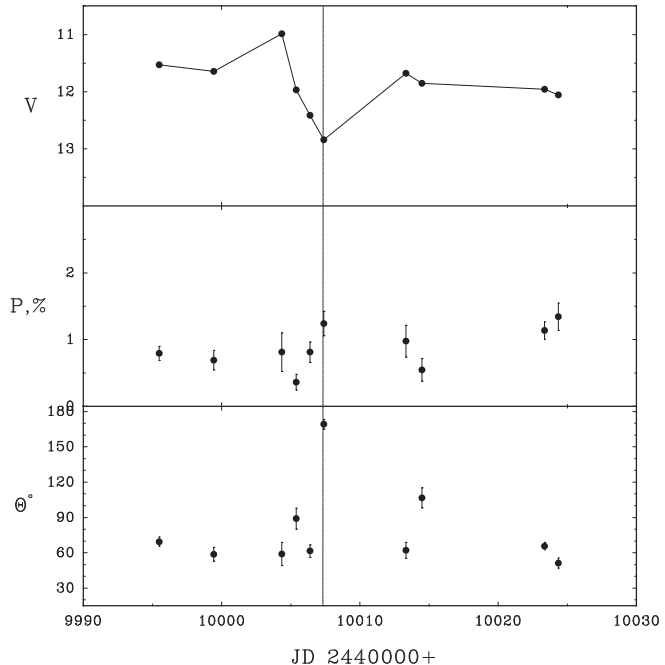

Fig. 5. Spike on the light curve, the negative flare of DF Tau at $\mathrm{JD}=2450007$, and its polarimetric response.

theoretical color tracks (Grinin 1980) and is caused by the increase in the optical thickness of the emitting gas beyond the Balmer jump at the increase in the gas temperature.

From the light curve presented in Fig. 2, we see that besides the strong, irregular brightness variability observed during each observational season, much slower variations also exist on a time scale on the order of ten years and more. Such variations are part of the longer photometric activity of DF Tau on a time scale of about $40 \mathrm{yr}$, revealed by Lamzin et al. (2001b) from analysis of the historical light curve of this star. In Sect. 4 we discuss different interpretations of this observational fact.

\subsection{Photopolarimetry}

The polarimetric variations of DF Tau are presented in Figs. 4-6. One can see that the parameters of linear polarization of the star fluctuate in the ranges $0.3 \leq P \leq 1.5 \%$ and $30^{\circ} \leq \theta \leq 170^{\circ}$. Both parameters do not show any dependence on the brightness changes. An exception is the deep minimum observed just after the flare-like event near JD 2450007 when the position angle $\theta$ changed strongly in comparison with the pre-flare values (Figs. 4-6). The degree of the linear polarization increased by a factor of two in the deepest part of the minimum in the comparison with the pre-flare state (Fig. 5). Such changes of intrinsic linear polarization are observed in UX Ori type stars (see e.g., Grinin et al. 1991; Rostopchina et al. 2000) and are explained by variable CS extinction on the line of sight.

Other interesting details of the polarimetric behavior of DF Tau include (Fig. 4): i) the shift of the position angle from $\theta \approx 110-120^{\circ}$ at JD ...9750 to $\theta \approx 30-50^{\circ}$ at JD ..10 160, and ii) the "jump" of $\theta$ between JD ..10160 and JD ..10350 by about $70-80^{\circ}$. Both of these details were not accompanied by sizable variations in the stellar brightness or degree of linear polarization. The characteristic time of these changes essentially 

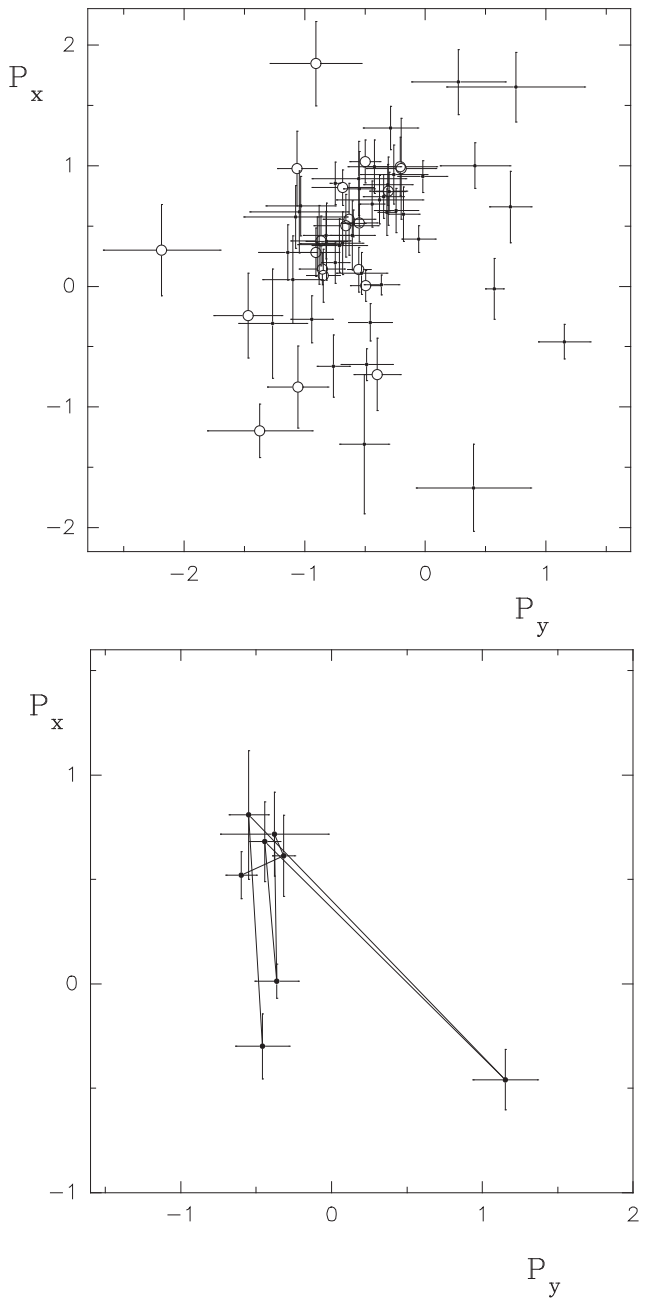

Fig. 6. Stokes parameters $P x-P y$ in $V$ band. The top panel corresponds to the data presented in Fig. 4 from JD $=2449750$ to $\mathrm{JD}=2450160$ (filled circles) and after the "jump" (open circles) of the position angle after JD $=2450150$; the lower panel corresponds to the data given in Fig. 5.

exceeds the rotation period of DF Tau of about 7 days (see e.g. Chelli et al. 1999) but is much less than the orbital period of the system. Therefore, these observations cannot be explained by changes in the illumination conditions in the system connected with the rotation of the spotted star or by changes in the dust environment caused by the orbital motions of the companions. It is possible that the above-mentioned changes in $\theta$ are the result of strong perturbations in the outer layers of the CS disk surrounding the primary (see Sect. 4).

The absence of the clear anti-correlation between the stellar brightness and degree of the linear polarization in all the photometric bands (Fig. 7) is an important observational fact. It indicates that the role of the variable circumstellar extinction in the photometric activity of DF Tau is not very important, although it may not be negligible.

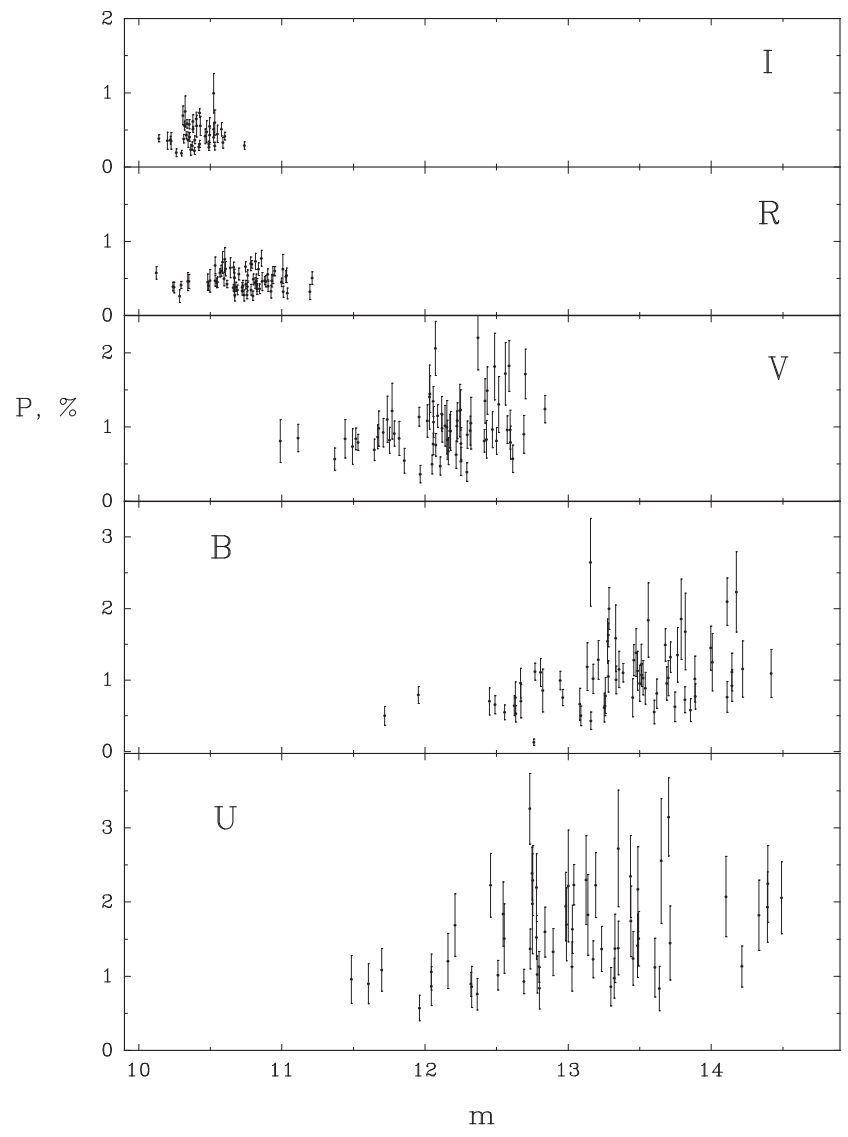

Fig. 7. Degree of the linear polarization in the different brightness states of DF Tau.

\subsection{Bispectrum speckle interferometry: brightness variations and orbit}

According to Table 3, the flux ratio $q$ of the main component to the secondary one in the $K$ band changed from $q=1.63 \pm 0.03$ in 2002.74 to $q=2.0 \pm 0.04$ in 2003.85. Comparison with the results of the previous observations by Chen et al. (1990), Ghez et al. (1997), and White \& Ghez (2001) shows that these values are typical of DF Tau.

Using our speckle interferometric observations of DF Tau (Table 3), together with the previous measurements of other authors summarized in the paper by Schaefer et al. (2003), we calculated the orbital parameters of this system using the apparent motion parameters method developed by Kiselev \& Kiyaeva (1980) and Kiselev et al. (1997). A solution was derived for a mean distance to Taurus of $140 \mathrm{pc}$ and for the total mass of the system $M=0.75 M_{\odot}$. The second value is very close to the sum of the mass of the system components estimated by Hartigan \& Kenyon (2003). They obtained the following values: $M_{1}=0.38 M_{\odot}$ and $M_{2}=0.35 M_{\odot}$. Agreement with all observations is obtained for the value of relative radial velocity of the components of $\Delta V_{r}=1.3 \mathrm{~km} \mathrm{~s}^{-1}$. If it were possible to obtain $\Delta V_{r}$ from observations, we could estimate the total mass of the system independently.

The new orbit is shown in Fig. 9, and its parameters are given in Table 4 . The previous estimates of the orbital parameters by Tamazian et al. (2002) (Model 2) are also given for 

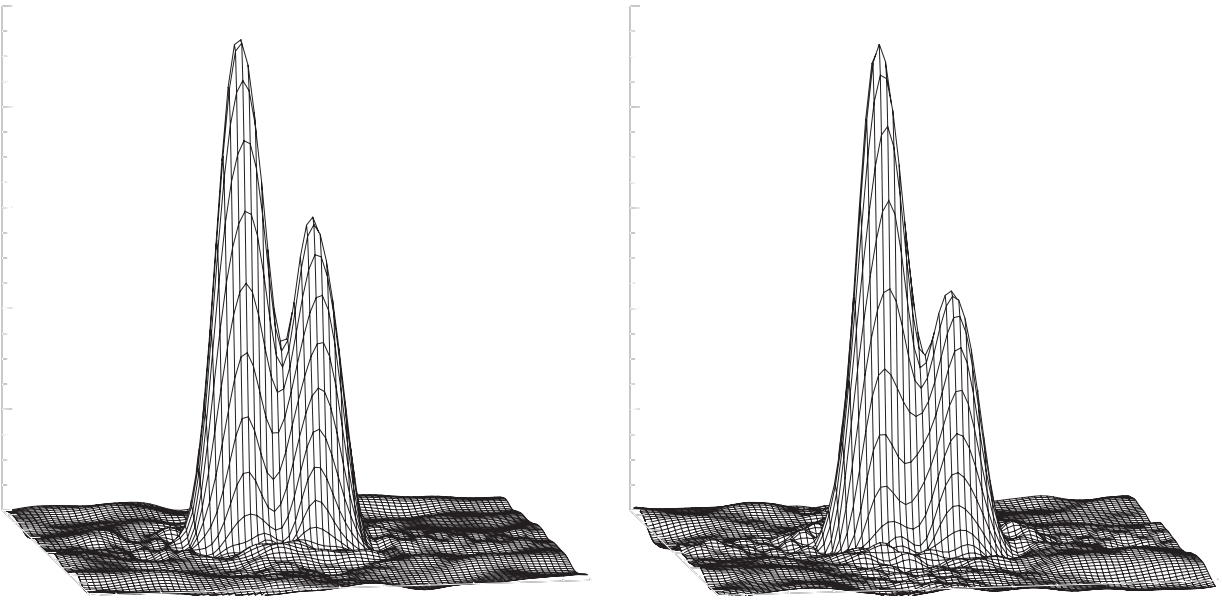

Fig. 8. $K$-band images of DF Tau in 2002 (left) and 2003 (right) obtained by bispectrum speckle interferometry.

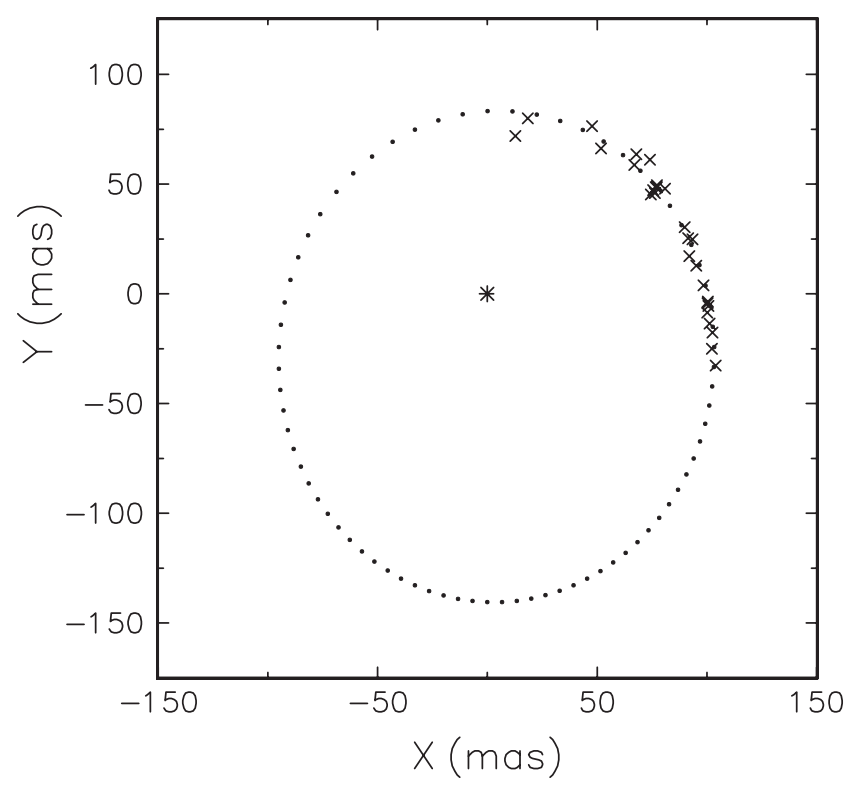

Fig. 9. New orbit of DF Tau. The observations from different sources made from 1986 to 2003 (see text) are shown by crosses.

comparison. It is important to note that the orbital parameters are quite sensitive to variations in the two initial parameters: the distance to the system and its total mass. For example, $10 \%$ uncertainty of the distance to DF Tau would give a $25 \%$ error in P. On the other hand, if we used $M_{\text {tot }}=1.0 M_{\odot}$ instead of 0.75 , we would obtain $P=55.5 \mathrm{yr}$ instead of 74.1 .

\section{Discussion}

Our observations suggest that the linear polarization of DF Tau does not depend on the brightness variation. This excludes the variable-extinction model as the main mechanism of the brightness variability since it predicts an anti-correlation between the degree of polarization and stellar flux (Grinin 1988). Nevertheless, as noted above, some episodes of the photometric activity of DF Tau cannot be explained without using this mechanism. Since the CS dust opacity increases as the wavelength decreases, the role of this mechanism should be greater in the blue part of the spectrum. This explains why the photometric histograms (Fig. 1) in the blue photometric bands have shapes which resemble those observed in the UX Ori type stars (Grinin et al. 1991).

It is natural to connect the relatively small influence of CS dust on the variability of DF Tau with the orientation of the CS disk around the primary. According to the current models (see e.g. Artymowicz \& Lubow 1996), the CS disk should be coplanar to the orbital plane. In this case the CS disk axis is inclined by 66 degree to the line-of-sight (see Table 4).

The absence of clear anti-correlation between the degree of polarization and stellar brightness also contradicts the model of a spotted star surrounded by a CS disk (Stassun \& Wood 1999). In the framework of this model, the physical reason for this anti-correlation is as follows: the hot accretion spot (a source of the non-polarized flux) can be behind the stellar disk, but its radiation scattered by the CS disk is observed. Therefore, only models with high-latitude spots or with small $i$ (when the star is seen close to pole-on) do not contradict the observed photopolarimetric activity of DF Tau.

The amplitude and color of the flare-like event near JD = 2450004 (Figs. 3, 4) are typical of CTTS (see e.g., Gahm et al. 1995; Gullbring et al. 1996). The most natural source of such flares is non-stationary, magnetically channelling accretion onto the star ${ }^{1}$. The fact that the flare was accompanied by the post-flare algol-type minimum suggests that both events were initiated by the same gas and dust stream.

Although the total duration of our photometric monitoring of DF Tau was about 20 years, we did not observe any flare during this time that would be similar to the very strong flare of DF Tau reported recently by Li et al. (2001). According to these authors, this flare had an amplitude of about 6 stellar magnitudes in the $B$ band. Its very unusual color in the brightness maximum $(B-V \approx-2.5)$ cannot be explained by any presently known radiation mechanisms. Taking into account the nonstandard method of observation for that flare (the spectroscopic observations without support of photometry), we believe that the flare parameters were determined incorrectly.

As discussed in Sect. 3.2, the position angle of the linear polarization of DF Tau changes in a very wide range. According to Fig. 6, the values of the Stokes parameters $P_{x}$

1 The recent measurements of magnetic fields in TTSs by Symington et al. (2005) give a polar field in DF Tau $\approx 4.5 \mathrm{kG}$. 
Table 4. Orbital parameters of DF Tau: model 1: new orbit; model 2: orbit reported by Tamazian et al. (2002).

\begin{tabular}{ccccccccc}
\hline \hline Model & $\begin{array}{c}P \\
(\mathrm{yr})\end{array}$ & $\begin{array}{c}M_{\mathrm{tot}} \\
M_{\odot}\end{array}$ & $\begin{array}{c}T \\
\mathrm{yr}\end{array}$ & $e$ & $\begin{array}{c}a \\
(\operatorname{arcsec})\end{array}$ & $\begin{array}{c}i \\
(\mathrm{deg})\end{array}$ & $\begin{array}{c}\Omega \\
(\mathrm{deg})\end{array}$ & $\begin{array}{c}\omega \\
(\mathrm{deg})\end{array}$ \\
\hline 1 & $74.1 \pm 8$ & 0.75 & $1986.0 \pm 2$ & $0.26 \pm 0.03$ & $112 \pm 0.01$ & $156.0 \pm 5$ & $353.0 \pm 8$ & $343.5 \pm 16$ \\
2 & $92.8 \pm 5$ & 0.82 & $1981.3 \pm 2$ & $0.51 \pm 0.04$ & $137 \pm 0.01$ & $135.1 \pm 3$ & $16.2 \pm 7$ & $326.4 \pm 10$ \\
\hline
\end{tabular}

and $P_{y}$ form a figure that is similar to an ellipse. Such behavior of the intrinsic linear polarization agrees well with the model predictions by Stassun \& Wood (1999), which predicts the strongest variability in the position angle for pole-on systems where the scattering plane changes a lot during a rotation period. This corresponds to our case since the expected inclination angle of the CS disk of DF Tau is small or intermediate: $i \approx 24 \pm 5^{\circ}$ (see above). An exception is the long-lasting trend of the position angle that was observed for about one year from $\mathrm{JD} \approx 2449750$ to $\mathrm{JD} \approx 2450160$ (Fig. 4). This time interval is too short in comparison with the orbital period and cannot be explained by the relative motions of the binary companions. The most likely reason for this polarimetric trend is a change in the geometry of the scattering region around the primary.

As mentioned above, the flux ratio of the binary components in the $K$ band mainly changes in the range of about 1.5 to 2.2. This variability is mainly caused by the primary (Ghez et al. 1997). The primary is also the main cause for the optical variability of the binary system DF Tau (Schaefer et al. 2003). The origin of such asymmetry is unclear. It could be the result of the strong inequality of the mass accretion rate onto the binary components, which is possible in young binary systems with low-mass secondary components (Artymowicz \& Lubow 1996; Bate \& Bonnell 1997). However, Johns-Krull \& Valenti (2001) and Hartigan \& Kenyon (2003) have shown that the masses of the DF Tau companions are very similar.

Another open question is the interpretation of DF Tau's photometric cycle of $\approx 40$ years (see Sect. 3.2). Our solution of the orbit (Table 4) gives a system period $\mathrm{P}$ of $74 \mathrm{yr}$, which is about twice the value of the cycle duration. However, the theory of young binary systems (Artymowicz \& Lubow 1996) predicts a modulation period of the accretion activity that is equal to the orbital period, and such a modulation is actually observed in the case of the well-studied young binary DQ Tau (Mathieu et al. 2000). Thus, the physical reason for the photometric cycle of DF Tau remains unclear.

\section{Summary}

Our observations suggest that the linear polarization of DF Tau does not depend on the brightness variation and that its changes have a stochastic character. This excludes the variable extinction model as the main mechanism of the brightness variability since it predicts an anti-correlation between the degree of polarization and stellar flux. At the same time, as noted above, some episodes of the photometric activity of DF Tau cannot be explained without using this mechanism. The relatively small influence of the CS dust on the variability of DF Tau is probably caused by the large inclination of the primary's CS disk to the line-of-sight.

The absence of anti-correlation between the polarization and brightness changes does not contradict the suggestion of the dominant role of hot accretion spots as a source of the photometric activity of this star. It suggests that the hot spots on the stellar surface are high-latitude spots and (or) the inclination of the stellar rotation axis to the line-of-sight is small or intermediate.

Using the total mass of the binary system DF Tau from Hartigan \& Kenyon (2003), we calculated the new orbital parameters of the system. The new value of the orbital period $P$ is $74.1 \mathrm{yr}$. It does not agree with the photometric cycle of about $40 \mathrm{yr}$ found by Lamzin et al. (2001). Therefore, it is possible that the photometric cycle is not caused by the orbital motions of the binary components, but that it reflects the cyclical variability of the global magnetic field on the main component.

Acknowledgements. We thank the referee, Pierre Bastien, for valuable comments and suggestions. This work was partly supported by the INTAS grant 03-51-6311 and the program "Non-stationary phenomena in Astronomy" of the Presidium of the Russian Academy of Sciences.

\section{References}

Artymowics, P., \& Lubow, S. H. 1996, ApJ, 467, L77

Basri, G., \& Batalha, C. 1990, ApJ, 363, 654

Bastien, P. 1982, A\&AS, 48, 153

Bastien, P. 1985, A\&AS, 59, 277

Bate, M. R., \& Bonnell, I. A. 1997, MNRAS, 285, 33

Bouvier, J., Bertout, C., \& Bouchet, P. 1986, A\&A, 158, 149

Bouvier, J., \& Bertout, C. 1989, A\&A, 211, 99

Bouvier, J., Grankin, K. N., Alencar, S. H. P., et al. 2003, A\&A, 409, 169

Chelli, A., Carrasco, L., Mújica, R., Recillas, E., \& Bouvier, J. 1999, A\&A, 345, L9

Chen, W. P., Simon, M., Longmore, A. J., Howell, R. R., \& Benson, J. A. 1990, ApJ, 357, 224

Gahm, G. F., Loden, K., Gullbring, E., \& Hardstein, D. 1995, A\&A, 301,89

Ghez, A. M., White, R. J., \& Simon, M. 1997, ApJ, 490, 353

Grinin, V. P. 1980, Astrophysics, 16, 147

Grinin, V. P., Kiselev, N. N., Minikhulov, N. Kh., Chernova, G. P., \& Voshchinnikov, N. V. 1991, Ap\&SS, 186, 283

Grinin, V. P. 2000, in Disk, Planetezimals \& Planets, ed. Y. F. Garzon, et al. ASPC 219, 216

Gullbring, E., Barwig, H., Chen, P. S., Gahm, G. F., \& Bao, M. X. 1996, A\&A, 307, 791

Gullbring, E., Hartmann, L., Briceño, C., \& Calvet, N. 1998, ApJ, 492, 323

Hartigan, P., Kenyon, S. J., Hartmann, L., et al. 1991, ApJ, 382, 617 
Hartigan, P., \& Kenyon, S. J. 2003, ApJ, 583, 334

Herbig, G. H., \& Bell, K. R. 1988, Lick. Obs. Bull., No. 111

Herbst, W., et al. 1994, AJ, 108, 1906

Hofmann, K.-H., \& Weigelt, G. 1968, A\&A, 167, L15

Johns, C. M., \& Basri, G. 1995, ApJ, 449, 341

Johns-Krull, C. M., \& Basri, G. 1997, ApJ, 474, 433

Johns-Krull, C. M., \& Valenti, J. A. 2001, ApJ, 561, 1060

Kenyon, S. J., Dobrzhycka, D., \& Hartmann, L. 1994, AJ, 108, 1872

Kiselev, A. A., \& Kiyaeva, O. V. 1980, Sovjet Astron. J., 57, 1227

Kiselev, A. A., Kiyaeva, O. V., \& Romanenko, L. G. 1997, in Visual double stars: formation, dynamics and evolutionary tracks, ed. J. A. Docobo (Kluwer Acad. Pub.), 377

Labeyrie, A. 1970, A\&A, 6, 85

Lamzin, S. A., Vittone, A. A., \& Errico, L., et al. 2001a, Astron. Lett., 27,313

Lamzin, S. A., Melnikov, S. Y., Grankin, K. N., \& Ezhkova, O. V. 2001b, A\&A, 372, 922

Li, J. Z., Ip, W. H., Chen, W. P., Hu, J. Y., \& Wei, J. Y. 2001, ApJ, 549, L89

Lohmann, A. W., Weigelt, G., \& Wirnitzer, B. 1983, Appl. Opt., 22, 4028

Mathieu, R. D., Ghez, A. M., Jensen, E. L. N., \& Simon, M. 2000, in Protostars and Planets. IV, ed. V. Mannings et al.

Menard, F., \& Bastien, P. 1992, AJ, 103, 564
Muzerolle, J., Calvet, N., \& Hartmann, L. 2001, ApJ, 550, 944

Parenago, P. P. 1954, Trudi Astron. Sternberg Inst., v. 25

Piirola, V. 1984, Observ. Astrophys. Labor. Univ. Helsinki Rept., 6, 151

Rostopchina, A. N., Grinin, V. P., Shakhovskoi, D. N., Thé, P. S., \& Minikulov, N. Kh. 2000, ARep, 44, 365

Rydgren, A. E., Zak, D. S., Vrba, F. J., Chugainov, P. F., \& Zaitseva, G. V. 1984, AJ, 89, 1015

Schaefer, G. H., Simon, M., Nelan, E., \& Holfeltz, S. T. 2003, AJ, 126, 1971

Stassun, K., \& Wood, K. 1999, ApJ, 510, 892

Symington, N. H., Harries, T. J., Kurosawa, R., \& Naylor, T. 2005, MNRAS, in press [arXiv: astro-ph/0501445]

Tamazian, V., Docobo, J., White, R., \& Woitas, J. 2002, ApJ, 578, 925

Thiebaut, E., Balega, Y., Balega, I., et al. 1995, A\&A, 304, L17

Unruh, Y. S., Collier Cameron, A., \& Guenther, E. 1998, MNRAS, 295, 781

Walker, M. F. 1987, PASP, 89, 1015

Weigelt, G. P. 1977, Opt. Commun., 21, 55

Weigelt, G., \& Wirnitzer, B. 1983, Opt. Lett., 8, 389

White, R. J., \& Gehz, A. M. 2001, ApJ, 556, 265

Wood, K., Kenyon, S. J., Whitney, B., \& Bjorkman, J. E. 1996, ApJ, 458, L79

Zaitseva, G. V., \& Lyutyi, V. M. 1976, Variable Stars, 20, 255 